identified. Quantitative and qualitative data show that the program was equally effective for Aboriginal and non-Aboriginal students. Furthermore, participants identified changes in their relationships with young people in the classroom subsequent to the program's implementation.

Conclusions: There is a need to ensure that resilience programs are adapted to enhance their cultural safety and relevance. To articulate the impact of adapted programs, mixed methodologies are essential.

\section{Autumn birth and the cognitive deficit schizophrenia subtype}

\section{Rock, A Jablensky, S Howell}

Clinical Research and Neurophysiology, Centre for Clinical Research in Neuropsychiatry, University of Western Australia, Perth, Australia

Background: Seasonal asymmetry of schizophrenia births is a well-documented phenomenon, with most studies finding a winter/early spring excess. This effect is consistent in the northern hemisphere but rare in the southern hemisphere. In Western Australia, using an endophenotype-based approach, we delineated a discrete, genetically distinct subtype of schizophrenia characterized by pervasive cognitive deficits (CDs), with the residual cases being cognitively spared (CS) (Hallmayer et al. 2005).

Purpose: We compared birth seasonality in Australianborn patients with $\mathrm{CD}$ and CS subtypes.

Methods: Data were grouped according to month of birth, with calendar adjustment. Season of birth was calculated using the Kuipers grouped method (Freedman 1979).

Results: The CD, but not the CS, subtype showed a significant season-of-birth effect, with an autumn peak (April) and a summer trough.

Conclusions: Analysis of northern hemisphere studies (Messias et al. 2004) found that the deficit syndrome of schizophrenia (Kirkpatrick et al. 2001), sharing some characteristics with our CD subtype, has a peak of summer births, at variance with the winter peak for all schizophrenia. Although the peak for CD is in autumn, it too contrasts with the spring peak of all schizophrenia births in Western Australia (Morgan et al. 2000). Despite numerous studies showing birth seasonality in schizophrenia, its cause remains elusive. Geographical differences in exposure to circannually fluctuating, nongenetic risk factor(s) may underlie this phenomenon (McGrath \& Welham 1999). A challenge for future studies is to map the seasonal relationship between schizophrenia endophenotypes and risk factors such as low birth weight.

\section{Using the semantic priming task in schizophrenia research: methodological and theoretical considerations}

\author{
A Stefanovic, S Rossell \\ Mental Health Research Institute, Melbourne, Australia
}

Background: Schizophrenia is characterized by disturbances in language and thought. Semantic priming (SP) paradigms have been frequently used to investigate language function in schizophrenia. The SP effect is the reaction time advantage that is achieved by priming a target to which a participant is responding with a semantically or associatively related word. In schizophrenia, this area of research has produced many contradictory results.

Methods: This is a comprehensive up-to-date review of research on SP in schizophrenia. It considers the significance of the specific task parameters used and the characteristics of the patient sample as possible reasons underlying discrepancies.

Results: From this review, it has been established that there are two robust variables that produce different results in schizophrenia. First, the relatedness proportion effect, where low proportions of related primetarget pairs result in reduced or normal SP in people with schizophrenia, while higher proportions lead to increased SP. Second, using indirectly related primetarget pairs results in increased SP in schizophrenia. Further, in terms of patient characteristics, patients with thought disorder produce the most consistently abnormal SP results.

Conclusions: The results indicate that enhanced automatic spread of activation might be one of the causes of language deficits in schizophrenia. In the framework of distributed network models, it is possible that the patterns of representations overlap more between different or unusual concepts in people with schizophrenia compared with healthy people, especially those with the symptom of thought disorder.

\section{Reduced dysbindin (DTNBP1) mRNA in hippocampus of patients with schizophrenia}

\section{CS Weickert', R Straub², J Kleinman², T Hyde², D Rothmond ${ }^{3}$}

'Neuroscience Institute of Schizophrenia and Allied Disorders (NISAD); ${ }^{2} \mathrm{CBDB}, \mathrm{NIMH}$; and ${ }^{3} \mathrm{MiNDS}$ Unit, NIMH, USA

Dysbindin has been implicated as an etiological factor in schizophrenia by genetic linkage studies, genetic association studies and molecular studies of postmortem brains of patients with schizophrenia. In this report, we 
tested if alterations in dysbindin mRNA were found in the hippocampus of patients with schizophrenia by using in situ hybridization. We found significantly reduced dysbindin mRNA in the dentate gyrus, CA4 and CA3, but not CA1, subregions of the hippocampus of patients with schizophrenia as compared with normal controls. Additionally, we found that dysbindin mRNA levels strongly and positively correlated with synaptophysin and spinophilin mRNA levels, which are known to be reduced in patients with schizophrenia. Our results suggest that reductions in dysbindin protein previously found in the hippocampus of patients with schizophrenia may be because of decreased dysbindin mRNA. The significant reduction of dysbindin mRNA found in the hippocampus confirms and extends our initial findings that dysbindin mRNA is significantly reduced in the frontal cortex and tends to be decreased in the midbrain of patients with schizophrenia (Weickert et al. 2004). Taken together, our results suggest that dysbindin mRNA reduction is not anatomically restricted, but may be anatomically specific, in the brains of patients with schizophrenia. Furthermore, subfield-specific reductions in dysbindin mRNA may lead to subfield-specific synaptic pathology in the hippocampus of patients with schizophrenic.

\section{The Stress Sampler Study II: psychological functioning and coping within a diabetes sample}

\section{T Showyin 1,2, K Baikie ${ }^{1,2}$, A Finch $^{1,2}$, P Mitchell', G Parker ${ }^{1,2}$, J Reddy ${ }^{1}$, PR Schofield ${ }^{3}$, L Wedgwood ${ }^{1,2}$, K Wilhelm ${ }^{1,4}$ \\ 'School of Psychiatry, University of New South Wales; ${ }^{2}$ The Black Dog Institute, Sydney, Australia; 'Prince of Wales Medical Research Institute; and \\ ${ }^{4}$ Consultation Liaison Psychiatry, St Vincent's Hospital, Sydney, Australia}

Background: Diabetes is a complex illness that involves ongoing monitoring and management. The stress of adjusting one's lifestyle to comply with the demands of the illness can often impact on an individual's mental health, therefore increasing the need for effective coping efforts. This paper aims to identify coping styles associated with positive psychological adjustment in a sample of hospital out-patients with diabetes mellitus.

Method: The Stress Sampler Study commenced in July 2006 and recruitment is ongoing at a hospitalbased diabetes clinic. The baseline measures include the Patient Health Questionnaire (which is based on DSM-IV diagnostic criteria), K-10 (psychological distress), SF-12 (physical and psychological self-report of functioning), clinical interview and the COPE (which measures a variety of coping responses).

Results and Conclusions: Preliminarily data will be presented on psychological distress and functioning and the association with current coping responses within this diabetic sample $(n>100)$. It is anticipated that these findings will contribute to our understanding of positive coping efforts associated with psychological adjustment in people with diabetes and may inform the education of diabetes management.

\section{[3H]Pirenzepine binding in the pathology of schizophrenia: studies in Brodmann's area 6}

\section{A Soulby, B Dean, E Scarr \\ Mental Health Research Institute, Melbourne, Australia}

Background: Changes in muscarinic receptors are widespread in the brains of subjects with schizophrenia, implicating them in the pathology of the disorder (Hyde \& Crook Chem Neuroanat 2001, 22 53-63). A previous study showed decreased levels of mRNA for the muscarinic M1 receptor in Brodmann's area (BA) 6 from subjects with schizophrenia (Mancama et al. Am J Med Genet B Neuropsychiatr Genet 2003, 119 $2-6)$. The purpose of our study was to further investigate this finding.

Methods: Using in situ radioligand binding and autoradiography, the density of [3H]pirenzepine binding was determined in BA 6 obtained postmortem from 20 subjects with schizophrenia and 20 subjects with no psychiatric history (controls).

Results: $[3 \mathrm{H}]$ Pirenzepine binding was differentially distributed in the cortical gray matter (inner layer = gray1; outer layer $=$ gray2). [3H]Pirenzepine binding was significantly lower in tissue from subjects with schizophrenia than in that from control subjects in both gray 1 (mean \pm SEM, $74.56 \pm 10.67$ vs. $118.80 \pm 5.47$ fmole/mgETE, $P<0.01)$ and gray2 layers $(102.90 \pm 14.09$ vs. $152.90 \pm 8.04$, $P<0.01)$. Furthermore, $[3 \mathrm{H}]$ pirenzepine binding was significantly different between layers in control subject tissue (gray1, $118.80 \pm 5.47$; gray2, $152.90 \pm 8.04 ; P<0.05)$, but not in that from subjects with schizophrenia (gray1, $74.56 \pm 10.67$; gray2 $102.90 \pm 14.09 ; P>0.05)$.

Conclusion: These results support previous findings that levels of M1 and/or M4 muscarinic receptors are significantly altered in cortical regions from subjects with schizophrenia, extending the regions affected to include BA6. 